\title{
Bericht über die Internationale wissenschaftliche Jubiläumskonferenz Angewandte Linguistik: Erfahrungen und Perspektiven, Poznań 16-17.10.2015
}

Vom 16.-17. November 2015 fand am Institut der Angewandten Linguistik der AdamMickiewicz-Universität zu Poznań (UAM) die Internationale wissenschaftliche Jubiläumskonferenz Angewandte Linguistik: Erfahrungen und Perspektiven statt. Der Anlass für diese wissenschaftliche Feier war der dreifache Jahrestag: im Studienjahr 2015/2016 feiert das Institut für Angewandte Linguistik, das bei seiner Gründung Lehrstuhl für Angewandte Sprachwissenschaften hieß, sein 50jähriges Bestehen. Auch seit 50 Jahren erscheint Glottodidacta. An International Journal of Applied Linguistics, die wissenschaftliche Zeitschrift des Instituts. Zugleich wurde dieses Jahr das zehnte Jubiläum der Umwandlung des direkten Vorläufers des Instituts - des Lehrstuhls für Glottodidaktik und Translatorik - in das heutige Institut für Angewandte Linguistik feierlich begangen. Die Jubiläumskonferenz wurde von den Mitarbeitern des Instituts mit dem Ziel veranstaltet, den Wissenschaftlern ein Forum zu bieten, auf dem Erfahrungen und Meinungen zur bisherigen Entwicklung der angewandten Linguistik ausgetauscht, sowie ihre Entwicklungstendenzen in Polen und im Ausland diskutiert werden konnten. Während der Konferenz wurden sowohl die Errungenschaften der Posener Schule der Angewandten Linguistik, als auch die Forschungsergebnisse anderer polnischer und ausländischer Forschungszentren auf dem Gebiet der Sprachwissenschaft, Translatorik, Fremdsprachendidaktik, Mehrsprachigkeit, der interkulturellen Kommunikation und Mediation, sowie der Literatur-, Medien- und Kulturdidaktik präsentiert. Zusammen beteiligten sich an der Konferenz über 140 Teilnehmer aus Polen und dem Ausland (Deutschland, aus den Niederlanden, Italien, Tschechien). Die Konferenz stand unter der gemeinsamen Schirmherrschaft des Rektors der Adam-Mickiewicz-Universität, Prof. Dr. habil. Bronisław Marciniak, der Dekanin der Neuphilologischen Fakultät, Prof. Dr. habil. Teresa Tomaszkiewicz und des Oberbürgermeisters der Stadt Poznań, Jacek Jaśkowiak.

Die feierliche Eröffnung der Konferenz fand in Collegium Minus, dem historischen Rektorat-Gebäude der Adam-Mickiewicz-Universität, statt. Prof. Dr. Andrzej Lesicki, Prorektor der Universität, Prof. Dr. habil. Teresa Tomaszkiewicz, Dekanin der Neuphlilologischen Fakultät und Prof. Dr. habil. Izabela Prokop, Direktorin des Instituts für Angewandte Linguistik skizzierten in ihren aufeinanderfolgenden Eröffnungsreden, wie sich das Institut im Laufe der Jahre entwickelte: es wurde an die Geschichte der Universität erinnert, dann der Platz des Instituts für Angewandte Linguistik in der Struktur der Neuphilologischen Fakultät beschrieben sowie derzeitige Stand des Instituts dargestellt. Prof. Dr. habil. Waldemar Pfeiffer, Gründer und langjähriger Leiter des Instituts für Angewandte Linguistik, erzählte von dessen Anfängen und erinnerte zudem an das wissenschaftliche Werk von Prof. Dr. habil. Ludwik Zabrocki, einem herausragenden Posener 
Sprachwissenschaftler, der 1965-1976 den Lehrstuhl für Angewandte Sprachwissenschaft innehatte. Unter den Gästen, die ihre Jubiläumsglückwünsche aussprachen, waren Prof. Dr. habil. Dr. h. c. mult. Franciszek Grucza, der Gründer des Instituts für Angewandte Linguistik der Warschauer Universität, Prof. Dr. habil. Stanisław Puppel, der Leiter des Lehrstuhls für Ekokommunikation der Adam-Mickiewicz-Universität, Prof. Dr. Andrzej Sitarski, Direktor des Instituts für Russische Philologie der UAM sowie Dr. Magdalena Wrembel im Namen der Dekanin der Englischen Fakultät der UAM, Prof. Dr. habil. Katarzyna Dziubalska-Kołaczyk.

Die Jubiläumskonferenz fand unter der wissenschaftlichen Leitung von Prof. Dr. habil. Izabela Prokop (Vorsitzende), Prof. Dr. habil. Waldemar Pfeiffer (Ehrenvorsitzender) und Mitgliedern in folgender Besetzung statt: Prof. Dr. Sylwia Adamczak-Krysztofowicz, Prof. Dr. Camilla Badstübner-Kizik, Prof. Dr. Joanna Kubaszczyk, Prof. Dr. Marcin Maciejewski, Prof. Dr. Alicja Sakaguchi, Prof. Dr. Barbara Skowronek, Prof. Dr. Aldona Sopata, Prof. Dr. Danuta Wiśniewska und Prof. Dr. Stephan Wolting. Das Organisationsbüro wurde von Prof. Dr. Sylwia Adamczak-Krysztofowicz und Dr. Augustyn Surdyk (Vorsitzende) geleitet und bestand aus den folgenden Mitgliedern: Dr. Magdalena Jurewicz, Dr. Joanna Kic-Drgas, Dr. Dorota Owczarek, Dr. Paweł Rybszleger, Dr. Katarzyna Trojan, Mag. Eva Teshajev, Dr. Anna Urban, Dr. Joanna Woźniak und Dr. Nadja Zuzok.

Zum Programm der Konferenz gehörten fünf Plenarvorträge, drei am ersten und zwei am zweiten Konferenztag. Den ersten Plenarvortrag hielt Prof. Dr. Dr. Csaba Földes, der über die Position, Entwicklungsdynamik und Förderung der deutschen Sprache weltweit im Blick der Angewandten Linguistik reflektierte. Im nächsten Plenarvortrag, den Prof. Dr. habil. Sambor Grucza hielt, wurde Angewandte Linguistik in Polen zwischen Tradition und Innovation beschrieben. Zum Abschluss dieses ersten thematischen Blocks fokussierte sich Prof. Dr. Fank G. Königs auf die Beschreibung des Beitrags der Angewandten Linguistik zur Erforschung des Lehrens und Lernens von Fremdsprachen. Den zweiten Konferenztag begann Prof. Dr. habil. Hanna Komorowska mit einem Plenarvortrag über Dilemmas der Fremdsprachenlehrer. Danach präsentierte Prof. Dr. habil. Stanisław Puppel das Konzept der Angewandten Linguistik als Manifestation von Endound Exo-Symbiose. Das Programm der Jubiläumskonferenz umfasste auch zwei Podiumsdiskussionen: eine am ersten Konferenztag zum Thema Perspektiven der Angewandten Linguistik weltweit unter Beteiligung von Prof. Dr. Bernhard Brehmer, Prof. Dr. Almut Hille, Prof. Dr. Bernd Müller-Jacquier und Prof. Dr. Norbert Nübler und eine am zweiten Tag zum Thema Perspektiven der Angewandten Linguistik in Polen (analog zum Motto der Konferenz) unter Beteiligung von Prof. Dr. habil. Andrzej Kątny, Prof. Dr. Beata Mikołajczyk, Prof. Dr. habil. Teresa Siek-Piskozub, Prof. Dr. Aldona Sopata und Prof. Dr. habil. Weronika Wilczyńska.

Im Rahmen der Konferenz wurden 79 Referate gehalten. Die thematischen Schwerpunkte der Vorträge umfassten die Gebiete der Sprachwissenschaft und der Translatorik, Fremdsprachendidaktik, Mehrsprachigkeit, der interkulturellen Kommunikation und Mediation sowie der Literatur-, Medien- und Kulturdidaktik, und nach dieser Klassifikation wurden sie in sechs Sektionssitzungen unterteilt: 
1. Sprachwissenschaft-Übersetzungswissenschaft (Leitung: Prof. Dr. habil. Izabela Prokop, Prof. Dr. habil. Alicja Sakaguchi, Dr. Paweł Kubiak, Dr. Paweł Rybszleger, Dr. Magdalena Jurewicz, Dr. Hanka Błaszkowska)

2. Glottodidaktische Aspekte der Mehrsprachigkeit (Leitung: Prof. Dr. Barbara Skowronek)

3. Linguistische und psycholinguistische Aspekte der Mehrsprachigkeit (Prof. Dr. Aldona Sopata, Prof. Dr. Danuta Wiśniewska)

4. Interkulturelle Sprachpädagogik [Glottopädagogik] (Prof. Dr. Sylwia AdamczakKrysztofowicz, Dr. Magdalena Aleksandrzak, Dr. Luiza Ciepielewska-Kaczmarek)

5. Interkulturelle Kommunikation (Prof. Dr. Stephan Wolting, Dr. Marta Janachowska-Budych, Dr. Anna Szczepaniak-Kozak)

6. (Medien-)Linguistische Sektion (Prof. Dr. Camilla Badstübner-Kizik, Prof. Dr. Joanna Kubaszczyk, Prof. Dr. Marcin Maciejewski, Dr. Agnieszka Poźlewicz, Dr. Anna Urban, Dr. Anna Pieczyńska-Sulik).

Die Ergebnisse der Konferenz lassen unter anderem die allgemeine Schlussfolgerung zu, dass die Perspektiven der Angewandten Linguistik weltweit und in Polen hochkomplex sind und dass ihr Untersuchungsgegenstand auf vielen wissenschaftlichen Gebieten weiter entwickelt und erweitert sein sollte.

Weiterführende Informationen zur Konferenz, zum Rahmenprogramm sowie Abstracts der Referate finden sich auf der Internetseite der Konferenz. ${ }^{1}$

Magdalena JASZCZYK, Anna MARKO Uniwersytet im. Adama Mickiewicza w Poznaniu

${ }^{1}$ Die Internetseite ist unter folgender Adresse zugänglich: www.ils.konferencja.org 